\title{
DEMONSTRATION OF ROBOTIC WHEELCHAIR IN FUKUOKA ISLAND-CITY
}

\author{
Yutaro Fukase \\ Space and Robot System Group \\ Institute of Technology \\ Shimizu Corporation \\ 4-17, Etchujima 3-chome, Koto-ku \\ Tokyo 135-8530, Japan \\ fukase@shimz.co.jp \\ Hitoshi Satoh \\ Space and Robot System Group \\ Institute of Technology \\ Shimizu Corporation \\ 4-17, Etchujima 3-chome, Koto-ku \\ Tokyo 135-8530, Japan \\ hitoshi_sato@shimz.co.jp \\ Keigo Takeuchi \\ Intelligent Space Project \\ Institute of Technology \\ Shimizu Corporation \\ 4-17, Etchujima 3-chome, Koto-ku \\ Tokyo 135-8530, Japan \\ takeuchikeigo@shimz.co.jp
}

\author{
Hiroshi Kanamori \\ Space and Robot System Group \\ Institute of Technology \\ Shimizu Corporation \\ 4-17, Etchujima 3-chome, Koto-ku \\ Tokyo 135-8530, Japan \\ kanamori@shimz.co.jp \\ Tetsuji Yoshida \\ Space and Robot System Group \\ Institute of Technology \\ Shimizu Corporation \\ 4-17, Etchujima 3-chome, Koto-ku \\ Tokyo 135-8530, Japan \\ yoshida.tetsuji@shimz.co.jp \\ Junichiro Maeda \\ Intelligent Space Project \\ Institute of Technology \\ Shimizu Corporation \\ 4-17, Etchujima 3-chome, Koto-ku \\ Tokyo 135-8530, Japan \\ jmaeda@shimz.co.jp
}

\begin{abstract}
We have been performing studies on the intelligent space to support various operations of mobile robots. This space will provide several functions, such as recognizing position of robots and objects in the area, searching proper route for robot to proceed, and controlling robots and other equipments. In order to conduct a practical study, we have developed a wheelchair-type mobile robot which has minimum functions for autonomous operations in the intelligent space, and conducted field experiments in public roads in Fukuoka Island-City residential area, where has been authorized as a 'Special Zones for Robot Development and Testing'. This paper will describe the experiments and results obtained..
\end{abstract}

Keywords: Robotic Wheelchair, Ultra Sonic Positioning System, Laser Range Finder, Radio Frequency Identification System

\section{INTRODUCTION}

In Japan, various kinds of service robots are required to respond to a serious problem of declining birthrate and aging society in our country. Helping human's activities, carrying objects and leading someone to destinations will be major tasks of these service robots, and trafficability in living space will be one of the most important functions for them. Installation of the moving systems including navigation, guidance and control subsystems into a single mobile robot may not be the best solution to realize service operations of the robot, because such a robot becomes too complicated and expensive. On the contrary, improvement of the surrounding infrastructures with IT equipments will be one of the practical solutions to realize harmless and reliable robot services.

Presently we have been studying on an intelligent space for mobile robots. As a part of this study, we have developed a simple mobile robot based on electric wheelchair and several demonstration tests were conducted on public roads in Fukuoka Island-City area.

\section{INTELLIGENT SPACE FOR MOBILE ROBOT}

We are studying intelligent space in which the autonomous operation of a robot is easily and safely realized. Even if the robot is unable to recognize surrounding objects by itself, the space around it figures out its position and path status, and navigates the robot to an adequate direction. Figure1 shows an application concept of the system we are proposing. Each sensor node detects the robots, obstacles and pedestrians in an area. The detected data are managed by a position server. Real-time path searching and navigating direction for robot are executed by a robot managing server. The building server activates elevators, doors, lights, etc according to the robot status. And these sensor nodes and servers are connected into a 


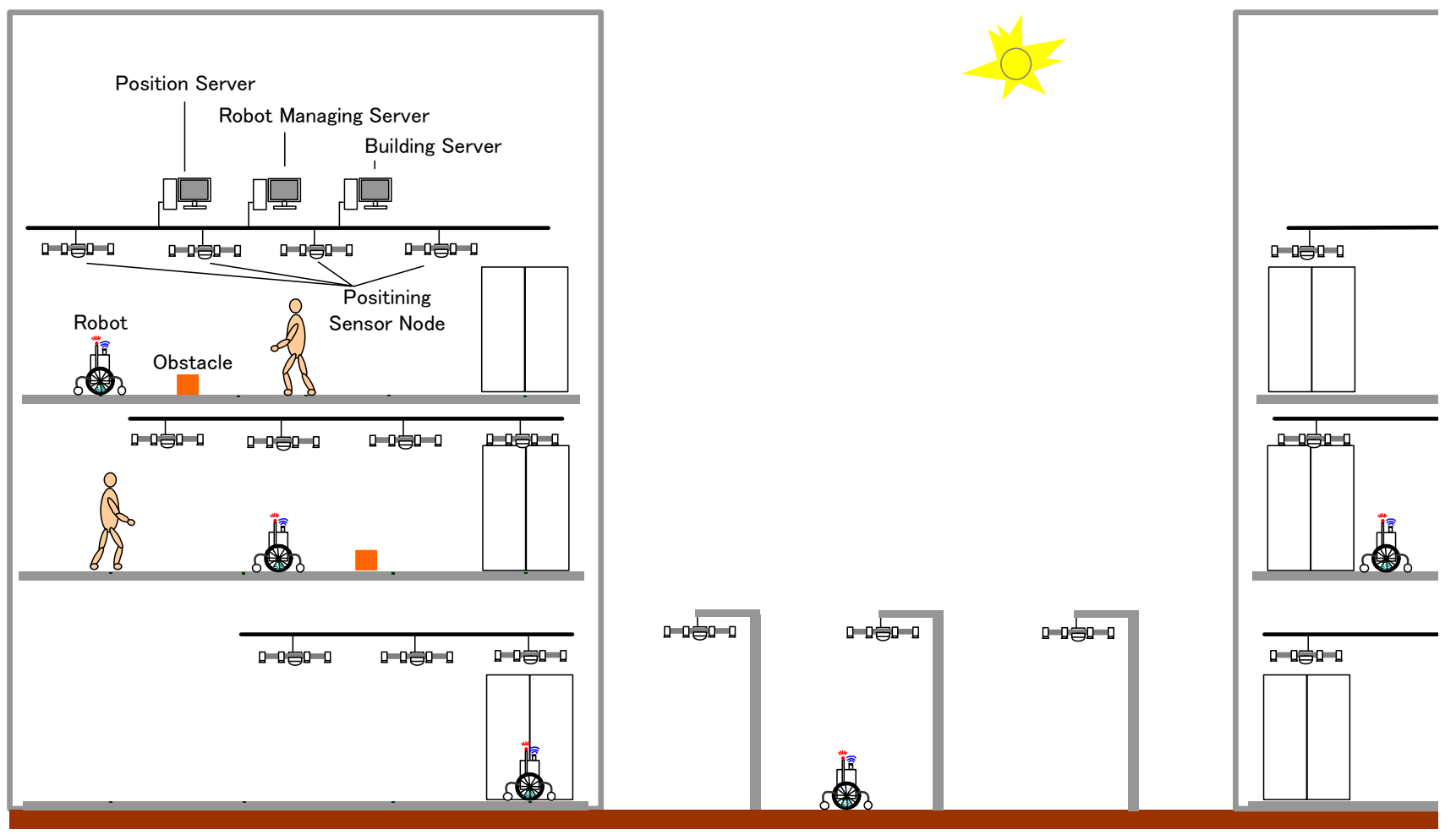

Figure 1. Intelligent Space for Mobile Robots

computer network and are working in a coordinated manner.

\section{THE ROBOTIC WHEELCHAIR}

\subsection{Feature of the Robotic Wheelchair}

In Japan, movements of barrier-free promotion make many public spaces easier for moving with wheelchairs. For example, numbers of sloops for wheelchairs along with stairs and pathways which are wider than wheelchair are drastically increasing. Users of electric wheelchair are also increasing and the price has been decreasing day by day. Thus we adopted a commercial electric wheelchair ${ }^{[1]}$ as a base system of the robot transferring mechanism.

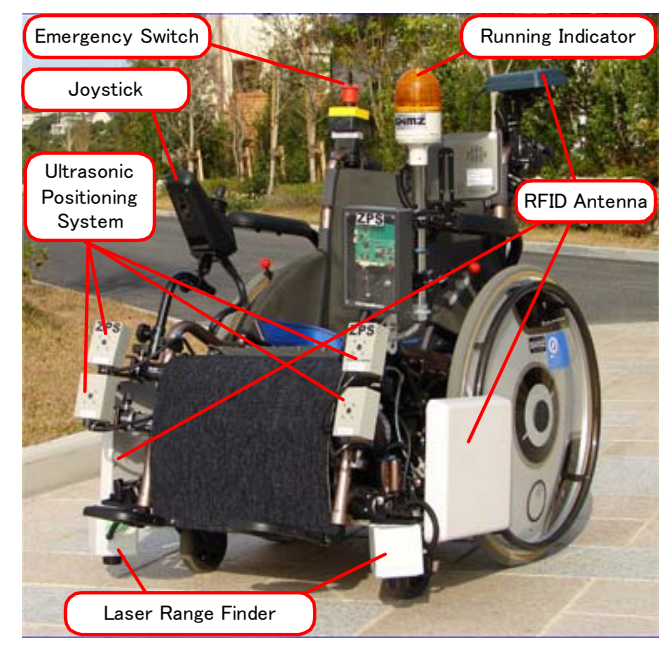

Figure 2. Robotic Wheelchair
Figure2 shows composition of the robot and Table1 shows specifications. System architecture of the robot is shown in Figure3.

Table 1. Specifications

\begin{tabular}{|l|l|}
\hline Dimensions & $1,100(\mathrm{~L}) \times 800(\mathrm{~W}) \times 1,100(\mathrm{H}) \mathrm{mm}$ \\
\hline Weight & $70 \mathrm{~kg}$ \\
\hline Power & Lead Battery (Max24V) \\
\hline Motor & $60 \mathrm{~W} \mathrm{DC} \times 2$ \\
\hline Max Speed & $4.5 \mathrm{~km} / \mathrm{h}$ \\
\hline Max Load & $50 \mathrm{~kg}$ \\
\hline \multirow{4}{*}{ Sensors } & Laser Range Sensor $\times 2$ \\
\cline { 2 - 2 } & Ultrasonic Positioning System \\
\cline { 2 - 2 } & RFID Reader \\
\hline \multirow{3}{*}{$\begin{array}{l}\text { Safty } \\
\text { Equipment }\end{array}$} & Emergency Switch \\
\cline { 2 - 2 } & Running Indicator \\
\cline { 2 - 2 } & Magnetic Break \\
\hline \multirow{3}{*}{$\begin{array}{l}\text { Moving } \\
\end{array}$} & Groping Mode \\
\cline { 2 - 2 } & Tailing Mode \\
\cline { 2 - 2 } & Odometry Mode \\
\cline { 2 - 2 } & Manual Mode \\
\hline
\end{tabular}

The robot has following four operational modes.

i ) Tailing Mode

The robot follows after a person's back with an ultrasonic positioning system.

ii) Automatic Mode

The robot gropes its way by detecting walls and gets commands from RFID-tags which are put on several points on the wall. 


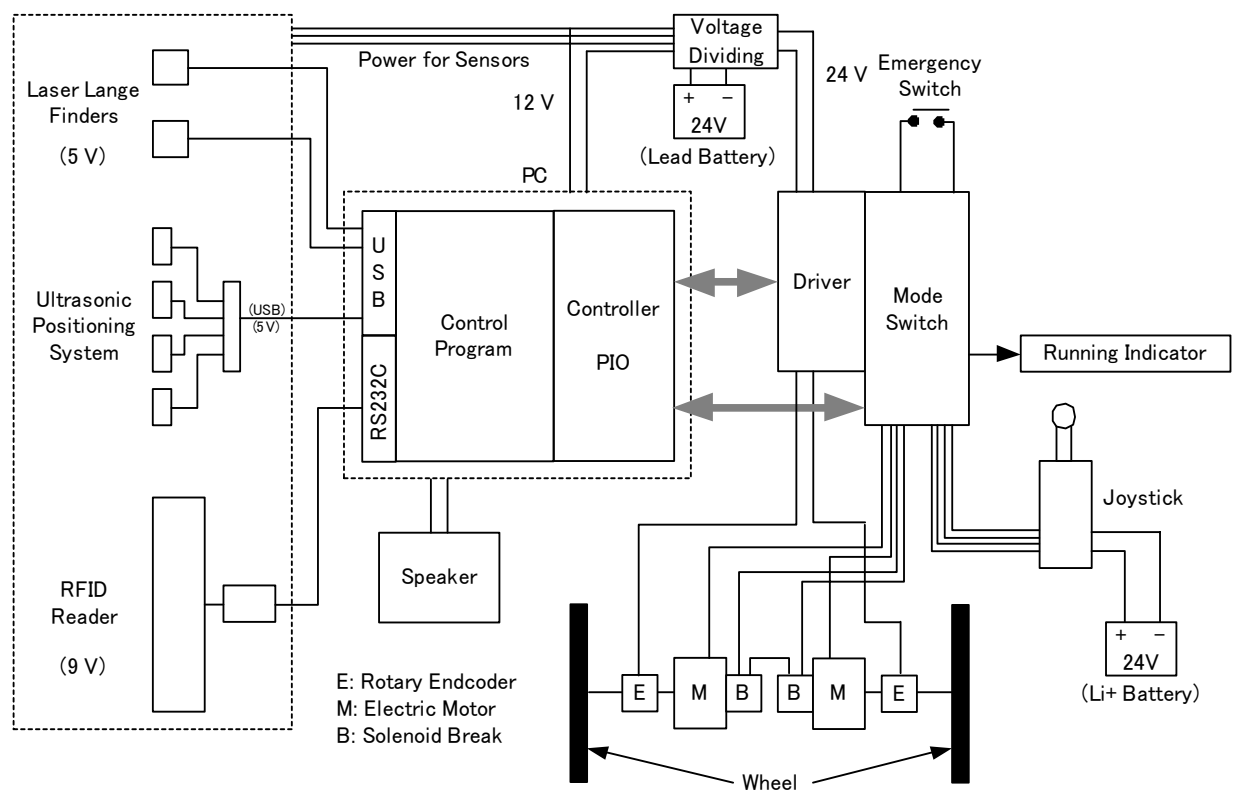

Figure3. Architecture of Robot

\section{iii) Odometry Mode}

The robot is navigated only by the wheel angle, which is counted with the encoder on the motor.

iv) Manual Mode

The robot is operated with a joystick on the right arm. This mode is an ordinary usage of the electric wheelchair.

\subsection{Tailing Mode}

\subsubsection{Ultrasonic Positioning System}

On the front side of the robot, four ultrasonic receivers and a radio communication unit are attached and connected to PC via an USB cable. In order to detect a specific ID tag which is attached to a person, the radio communication unit sends a command to the tag by radio wave. When the tag receives that command, it starts to sound ultrasonic beacon. The four ultrasonic receivers on the robot listen that sound and measure the time of flight from the tag to each receiver and calculate the position of the tag. This system is supplied by Furukawa CO., LTD. ${ }^{[2]}$

\subsubsection{Control for Tailing Tag}

We control the robot as a model in which two pairs of spring and dumper are connected to its right and left sides (Figure5). The generated force on each wheel depends on this model excepting in a case in which a person approaches to the robot. In that case, both left and right virtual springs are shorter than natural length, and then we only activate the dumper forces to stop the robot.

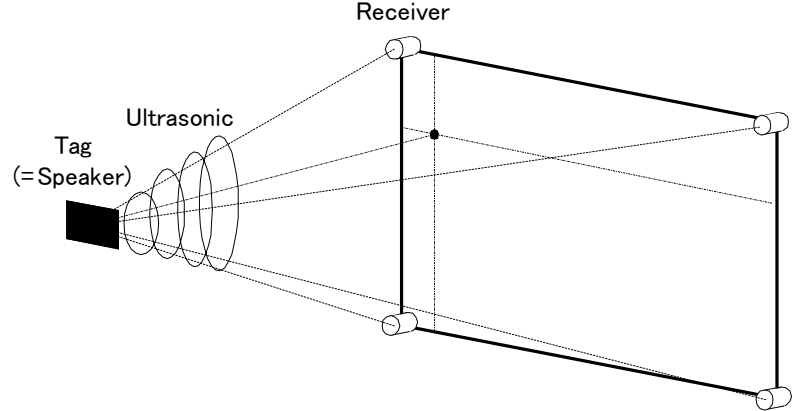

Figure 4. Ultrasonic Positioning System

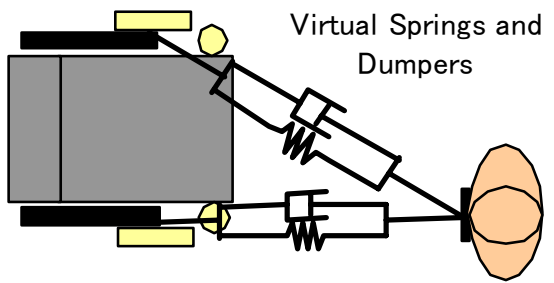

Figure 5. Control of Tailing Tag

\subsection{Automatic Mode}

\subsubsection{Detection of Wall}

Two laser range finders are located near each right and left footrest. Each sensor measures a distance from the sensor to an object. Its measuring range is from $20[\mathrm{~mm}]$ to $4000[\mathrm{~mm}]$ in distance and 270 [deg] in field angle. Its accuracy is within $1[\%]$ and the resolution of searching angle is $0.36[\mathrm{deg}]$. The computer searches a shortest point in the data cloud and crops the points near a line perpendicular to a line drawn form the sensor to the shortest point. Then it figures out a wall as collinear approximation 
on the cropped points. This laser range finder was supplied by Hokuyo Automatic CO., LTD. ${ }^{[3]}$

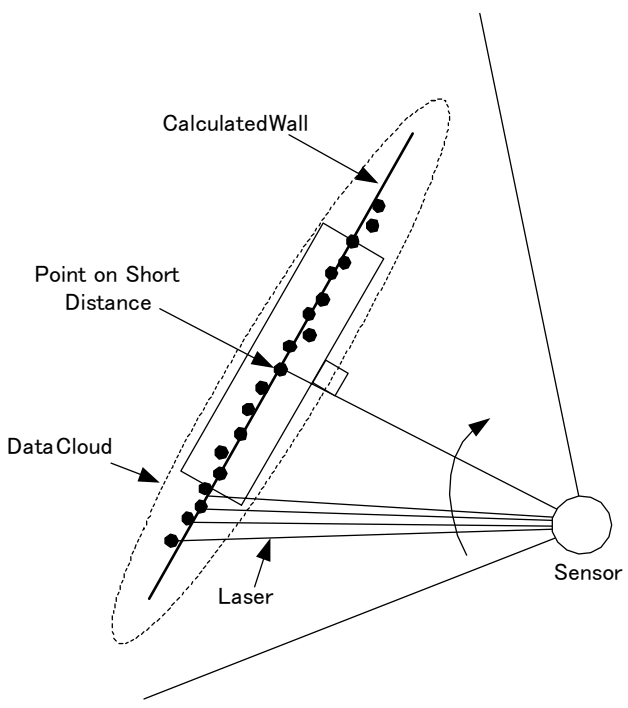

Figure 6. Calculating Wall Position

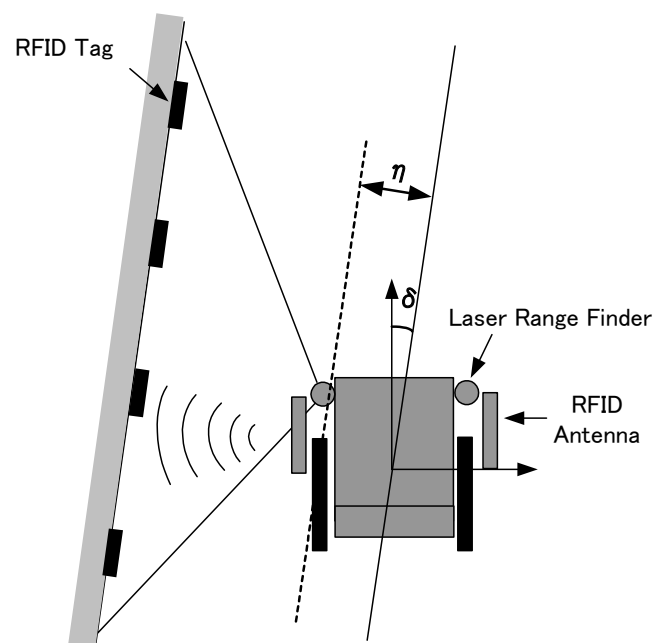

Figure 7. Control of Groping Wall

\subsubsection{Control of Groping Wall}

A forward speed of the robot and a target distance between the wall and the robot are first set. In order to make a moving direction of the robot parallel to that of the wall, and to make the distance between the wall and the robot at the set value, the reference body angle speed $\omega_{\text {ref }}$ is calculated from the following equations.

$\Delta \omega / \Delta t=K 1 \times \delta+K 2 \times \omega_{\text {now }}+K 3 \times \eta$

$\omega_{\text {ref }}=\omega_{\text {now }}+\Delta \omega / \Delta t \times \Delta t$

$K 1, K 2, K 3$ : set coefficient

$\delta$ : deviation of angle

$\omega_{\text {now }}$ :present body angle speed

$\eta$ : deviation of distance

$\Delta t:$ controlling interval
A rotating speed of each right and left side wheel is calculated from the following equation.

$$
\begin{aligned}
& V=\text { Const } \\
& \omega_{\text {Left }}=\left(V-L \cdot \omega_{\text {ref }}\right) / R \\
& \omega_{\text {Right }}=\left(V+L \cdot \omega_{\text {ref }}\right) / R \\
& V: \text { set body forward speed } \\
& \omega_{\text {Left }} \text { :left wheel rotation speed } \\
& \omega_{\text {Right }} \text { :right wheel rotation speed }
\end{aligned}
$$

$L$ : half length of distance between right and left wheel $R$ :radius of wheel

\subsubsection{Detection of Obstacles}

Using the laser range finders, the PC detects obstacles around the robot as well as the walls. The right and left range clouds are merged for this purpose. When an object is detected in the slow down zone, the robot decreases its forward speed $(=\mathrm{V})$. When an object is detected in the stop zone, each motor generates a commeasurable negative force according to its rotating speed.

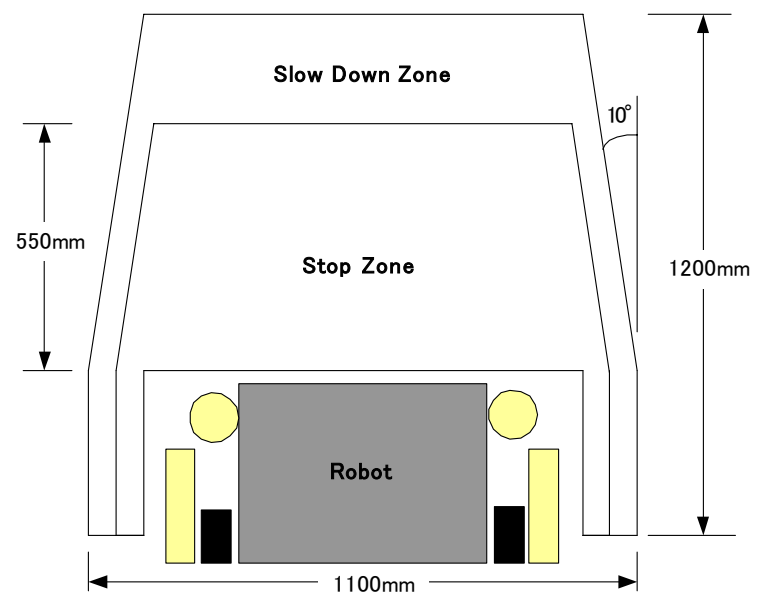

Figure 8. Range of Detecting Obstacle

\subsubsection{RFID Instruction}

In order to read RFID-tags located on several points on the wall, two antennas were attached on each side of the robot. A smaller antenna was also attached on a handle backside of the robot. Operator can touch the smaller antenna with a tag in his hand and easily instruct the robot. Reading interval is $250 \mathrm{~ms}$, and a motion of the robot such as stop, turn right, turn left, turn 180 degrees or changing moving mode, etc. is selected according to the corresponding ID of the tag.

\section{DEMONSTRATION EXPERIMENT}

4.1 Location and Date
a. Location 
Several experiments were carried out at a residential zone in Fukuoka Island-City, which is an artificial island in Hakata bay, Fukuoka city. The city is intensely promoting industrial renovation with robotics, and actively invites robotics competitions such as 'ROBOCUP'. In 2003, Fukuoka was designated as a "Special Zones for Robot Development and Testing" by the Japanese national government. And robot testing is now allowed on public streets ${ }^{[4]}$.

\section{b. Date}

Demonstration experiments were held three times, in September, October and November, 2005.

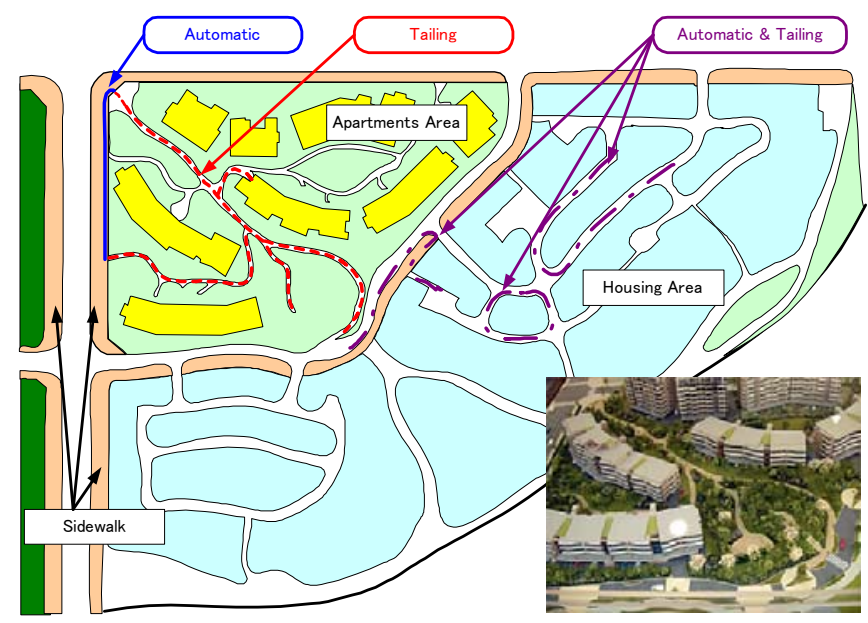

Figure 9. Residential Zone in Island City

\subsection{Experiment Description}

Evaluated items are listed as follows.

1) Tailing Mode

- Dynamic response for person's actions, such as walking, stopping and turning

- Detecting performance for person's position

- Moving performance on sloops and bumping pathways

2) Automatic Mode

- Groping wall performance

- Dynamic response for detected walls and obstacles

- Reading RFID performance and behavior trigged by RFID

\subsection{Result}

\section{1) Tailing Mode}

In the first experiment, the robot could not tail to a person at more than $2[\mathrm{~km} / \mathrm{h}]$. The reasons were supposed to be the noises of sound generated by strong wind, radio disturbances, and the low limit of wheel speed. Then in the second experiment, both of the sound level of ultrasonic positioning system, and the limit of the wheel speed were increased., As a result, the performance of tailing a person (=tag) became better. However, the stable detection of the tag was still not realized.
In Figure10, blue rhombic plots show the tag positions detected by the ultrasonic positioning system in front of the robot. The robot tailed the person well in the range from $50[\mathrm{~cm}$ ] to $3[\mathrm{~m}]$, and between -10 [deg.] to +10 [deg.].

When nobody sat on the chair, the robot moved smoothly in many cases. On the several bumps and sloops, where it needed larger torque, the wheel stopped by an overload error. This is caused by the poor performance of motor driver. It will be able to be improved by changing motor driver device.

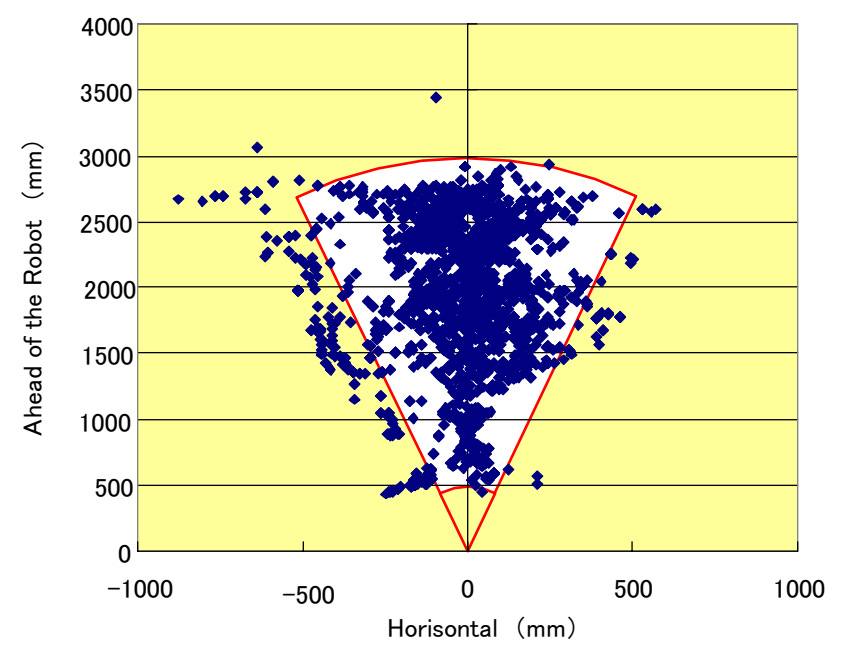

Figure 10. Range of Tailing Person

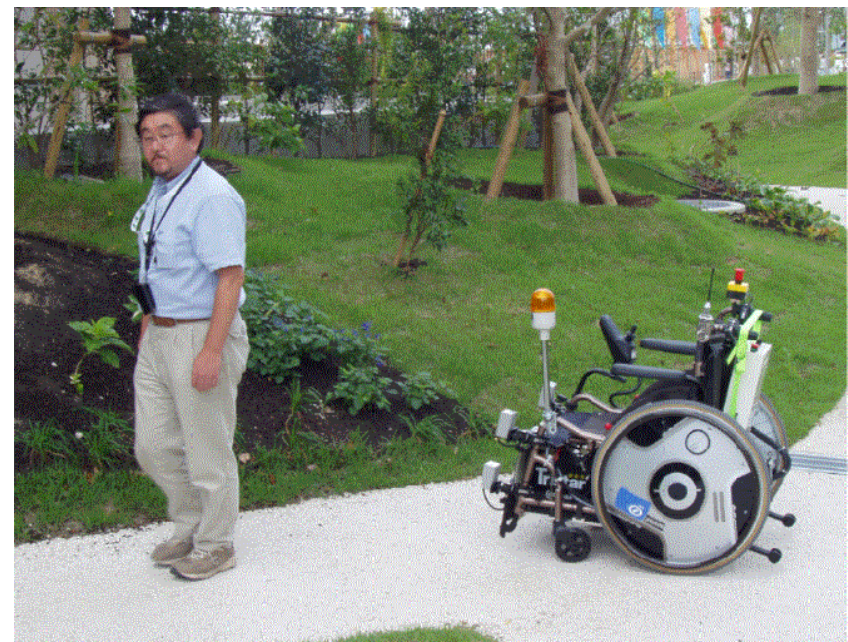

Figure 11. Demonstration of Tailing Mode

\section{2) Automatic Mode}

The robot could grope walls, curbstones and parcloses in almost all cases (Figure 12). It occasionally happened that a leaf of a plant near wall (Figure 13) was detected as an obstacle and made the robot to stop.

RFID-tags were located on the point where the robot had to turn back, left or right. When the robot read the tag, it executed a preprogrammed task, which corresponds to the tag's ID.

In front of a garage of a house, there was no wall or curbstones (Figure14). In order to move this area continuously, the robot, trigged by a RFID-tag, switched the 

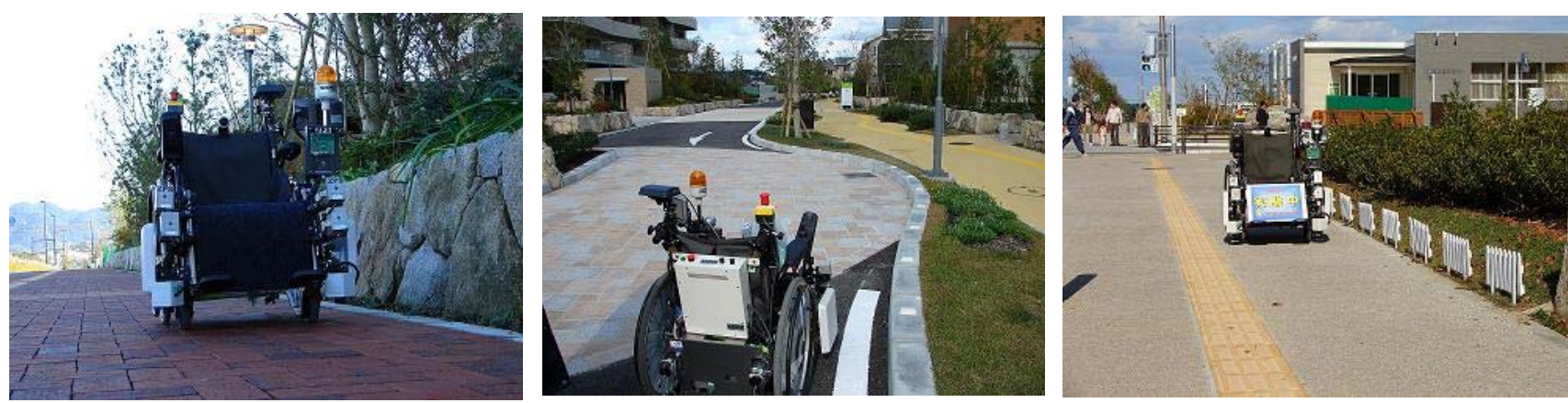

Figure12. Groping Wall, Curbstone and Parcloses

moving mode from the automatic mode to the odometry mode. Where the road in front of the garage was even or short, the robot could move stably to the next wall and then switched to the automatic mode again. When there was an unexpected unevenness or a manhole like object, the robot changed direction and could not reach the next wall. Installation of internal motion sensors, such as gyroscopes, compasses and accelerometers was expected to solve this problem.

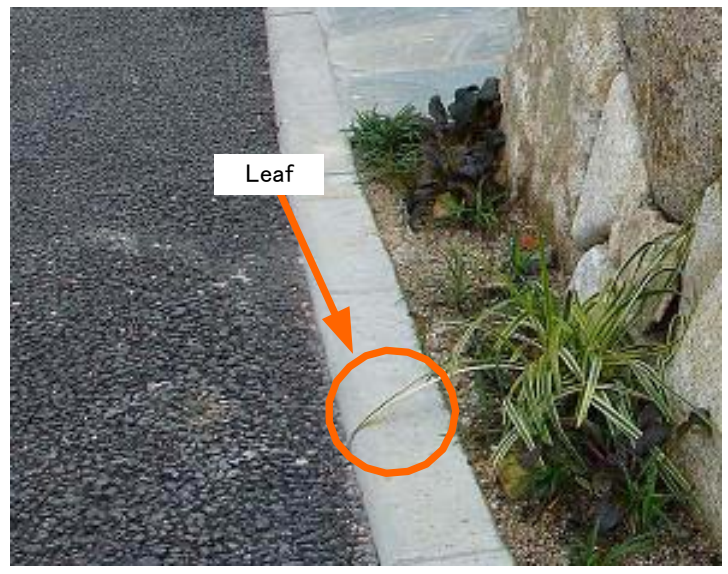

Figure13. Leaf detected as Obstacle

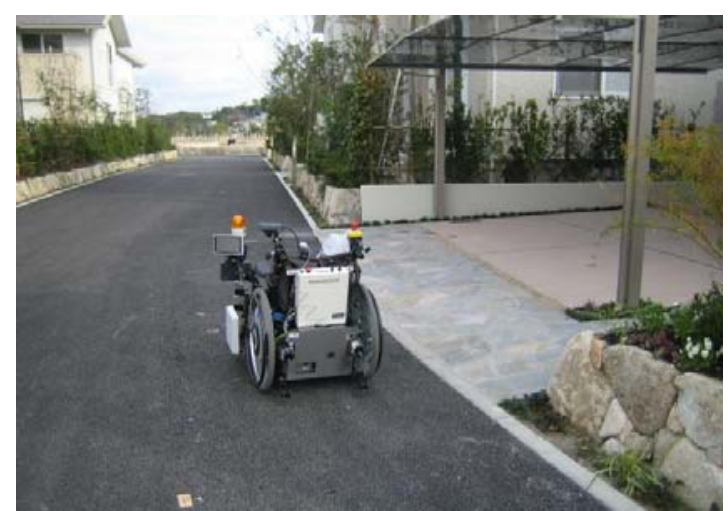

Fgiure14. Demonstration of Odometry Mode

\section{SUMMERY}

In order to conduct a study on intelligent space where robots can move easily, safely and stably, we have developed a mobile robot as a portion of the whole system.

Using a combination of the automatic mode, tailing mode and odometry mode, the robot was able to move on public streets. In these experiments, we encountered several problems on a robotic wheelchair, and obtained various ideas to solve these problems.

The fundamental concept of our intelligent space will be applied not only to the wheelchair systems, but also to the other systems, such as transportation in medical institutions and customer support in shopping areas.

\section{ACKNOWLEDGMENTS}

We express our thanks to the stuffs in Fukuoka-City and Sekisui House Corporation, who gave us a chance to have a demonstration experiments in Island City and cooperated with this R\&D.

\section{REFERENCES}

[1] URL about TAO-Light II, Aisin Seiki Co. http://www.keepable.net/product/wheelchair /index.html

[2] URL about ZPS, Furukawa Co. http://www.furukawakk.jp/products/ZPS_1.html

[3] URL about URG-04LX, Hokuyo Automatic Co. http://www.hokuyo-aut.jp/products/urg/urg.htm

[4] URL about Fukuoka Island-City, Fukuoka City http://www.island-city.net/business/it/it.html 